(c) American Dairy Science Association, 2006.

\title{
Evaluation of Environmental Sampling and Culture to Determine Mycobacterium avium subspecies paratuberculosis Distribution and Herd Infection Status on US Dairy Operations
}

\author{
J. E. Lombard, ${ }^{*} \dagger^{1}$ B. A. Wagner, ${ }^{*}$ R. L. Smith,$\ddagger^{2}$ B. J. McCluskey, ${ }^{*}$ B. N. Harris, $\S$ \\ J. B. Payeur,§ F. B. Garry,† and M. D. Salman\| \\ *USDA, Animal and Plant Health Inspection Service (APHIS), Veterinary Services (VS), Centers for Epidemiology and Animal Health, \\ 2150 Centre Ave., Bldg. B, Fort Collins, CO 80526-8117 \\ †Integrated Livestock Management, Colorado State University, Fort Collins 80523 \\ $\ddagger$ Cornell University, College of Veterinary Medicine, Ithaca, NY 14853 \\ §USDA, Animal and Plant Health Inspection Service (APHIS), Veterinary Services (VS), National Veterinary Services Laboratories, \\ 1800 Dayton Ave, Ames, IA 50010 \\ |Animal Population Health Institute, Colorado State University, Fort Collins 80523
}

\section{ABSTRACT}

The objectives of this study were to determine the distribution of Mycobacterium avium subspecies paratuberculosis (MAP) in the environment and assess the relationship between the culture status of MAP in the farm environment and herd infection status. The National Animal Health Monitoring System's Dairy 2002 study surveyed dairy operations in 21 states. One component of the study involved collection and culturing of environmental samples for MAP from areas on farms where manure accumulated from a majority of a herd's cows. Operations were selected for inclusion based on perceived risk factors for MAP infection identified in a previously administered questionnaire. Individual animal and environmental samples were collected and used to determine the efficiency of environmental sampling for determination of herd infection status. Individual animal fecal, serum, and milk samples were used to classify herds as infected or not infected based on the presence of at least one test-positive animal in the herd. A total of 483 environmental samples (approximately 5 per farm) were collected, and 218 (45.1\%) were culture-positive for MAP. A similar percentage of environmental cultures collected from all designated areas were positive [parlor exits $(52.3 \%)$, floors of holding pens $(49.1 \%)$, common alleyways (48.8\%), lagoons (47.4\%), manure spreaders (42.3\%), and manure pits (41.5\%)]. Of the 98 operations tested with the environmental sample culture, 97 had individual serum ELISA results, 60 had individual fecal culture results, and 34 had individual milk ELISA results. Sixty-nine of the 98 operations (70.4\%) had at least one environmental sample that was culture-positive. Of

Received April 13, 2006.

Accepted May 26, 2006

${ }^{1}$ Corresponding author: jason.e.lombard@aphis.usda.gov

${ }^{2}$ Current affiliation: Kansas State University, Manhattan. the 50 herds classified as infected by fecal culture, 38 $(76.0 \%)$ were identified by environmental culture. Two of the 10 operations classified as not infected based on individual animal fecal culture were environmental culture-positive. Of the 80 operations classified as infected based on serum ELISA-positive results, 61 (76.3\%) were identified as environmental-positive, whereas 20 of the $28(71.4 \%)$ operations identified as infected based on milk ELISA were detected by environmental sampling. Environmental sample culturing is less costly than individual animal sampling, does not require animal restraint, and identified more than $70 \%$ of infected operations. Environmental sampling is another diagnostic tool that veterinarians and dairy producers can use to determine herd infection status for MAP.

Key words: environmental sampling, paratuberculosis, dairy

\section{INTRODUCTION}

Johne's disease (JD), or paratuberculosis, is a chronic, progressive disease of ruminants caused by the effects of Mycobacterium avium subspecies paratuberculosis (MAP) infection on the gastrointestinal tract. It is transmitted primarily through a fecal-oral route, especially from manure and environmental contamination by infected adult cattle, and young cattle are most susceptible (Sweeney, 1996). The disease is manifested in adult cattle, leading to economic losses associated with decreased milk production, early culling, and decreased carcass weight. Control of this disease has become a focus of programs in several countries (Sockett, 1996; Kennedy and Benedictus, 2001).

In recent years, many JD control programs have focused on the use of pooled fecal cultures to screen herds for MAP infection status. For small herds with a low prevalence and large herds with a high prevalence, pools of 5 to 10 samples/pool have proved to be valid and cost- 
effective (Kalis et al., 2000; Wells et al., 2002; van Schaik et al., 2003; Wells et al., 2003; Tavornpanich et al., 2004). However, this method has not been appropriately sensitive for medium to large dairy herds with a low prevalence of MAP infection (Wells et al., 2002). The pooled fecal culture method requires individual cow sampling and can vary in sensitivity based on the stage of disease and the shedding level of animals sampled.

The presence of MAP in the dairy environment is believed to be a risk factor for the spread of MAP through a herd. Mycobacterium avium subspecies paratuberculosis has been shown to survive in manure slurry long-term at temperatures approaching freezing (Jorgensen, 1977). A more recent study has shown increased duration of MAP survival in shaded soil and pasture (Whittington et al., 2004). Lack of shade was shown to decrease survival, presumably because of infrared wavelengths and temperature flux. This evidence suggests that MAP survives for long periods of time in common dairy farm environments. However, the distribution of MAP in the dairy environment is not well documented. Samples collected from areas on dairy operations where manure accumulates from a majority of adult animals are commonly referred to as environmental samples. A study conducted in Minnesota by Raizman et al. (2004) found the most common areas to obtain culture-positive samples were cow alleyways and manure storage areas. Environmental sampling detected $78 \%$ of known infected herds identified by previous testing and $95 \%$ of herds in which the concurrent pooled fecal culture was positive. Berghaus et al. (2006) performed a similar study on 23 California dairies and found that lagoon water was the most likely to yield a positive culture and that environmental sampling identified 65 to $74 \%$ of infected operations identified by previous or concurrent individual animal testing. Further understanding of the relationship between MAP distribution, the environmental culture method, and other standard screening tests would be useful in further developing the environmental culture as a herd-level screening method.

The objectives of this study were 1) to determine the distribution of MAP in the environment and herd characteristics associated with culture-positive environmental samples on US dairy farms and 2) to assess the relationship between culture status of MAP in the farm environment and herd infection status as determined by individual cow fecal culture, serum ELISA, and milk ELISA.

\section{MATERIALS AND METHODS}

\section{Herd Selection}

States included in the National Animal Health Monitoring System Dairy 2002 study were selected to represent at least $70 \%$ of the animal and producer populations in the United States. Data from the USDA, National Agricultural Statistics Service (NASS), were used to determine the major US dairy states based on animal populations. Operations were selected from a stratified random sample of the NASS list frame to participate in phase I of the study, whereas those operations that had completed phase I (which consisted of a questionnaire), had 30 or more dairy cows, and were willing to continue to participate were included in phase II. Final selection for phase I included operations from 21 states from 4 regions of the United States and represented $83.0 \%$ of dairy cattle operations and $85.7 \%$ of dairy cattle (USDA, 2002). Data collected from phase I included herd size ( small $=$ less than 100 cows, medium $=100$ to 499 , and large $=500$ or more), region and state $($ West $=$ California, Colorado, Idaho, New Mexico, Texas, and Washington; Midwest = Illinois, Indiana, Iowa, Michigan, Minnesota, Missouri, Ohio, and Wisconsin; Northeast $=$ New York, Pennsylvania, and Vermont; Southeast = Florida, Kentucky, Tennessee, and Virginia), breed, rolling herd average (RHA) milk production, and housing type (multiple, individual, and none). Information on herd characteristics, operation history of JD (including animals with clinical signs, defined as chronic diarrhea and weight loss that did not respond to treatment despite a normal appetite), and previous testing were collected in phase II.

A subset of herds participating in phase II were selected to participate in environmental sampling and within-herd MAP prevalence testing-using fecal culture and serum ELISA-based on the operations' perceived risk of having and transmitting MAP. The operations eligible for phase II were then ranked from high to low risk based on perceived risks factors from their answers from phase I and included 1) number of dairy cows, 2) how soon calves were separated from their dams, 3 ) whether pooled colostrum was fed, 4) the percentage of dairy cows that had diarrhea for more than $48 \mathrm{~h}, 5$ ) whether maternity housing was separate from lactating cow housing, 6) how many replacements were brought onto the operation during 2001, and 7) whether the operation required JD testing for purchased cattle. Perceived low- and high-risk herds were identified from each participating state, with herds stratified into below and above median herd size for the state. Between 4 and 6 operations were selected in each participating state. Two operations were chosen from the low-risk group-one above and one below the median herd size for the state. Three operations were chosen from the high-risk groupeither 2 above the median herd size and one below the median herd size, or vice versa. From the operations selected, a further subset of herds that participated in DHIA testing was also asked to participate in milk ELISA testing. 


\section{Animal Selection}

Cows in the second lactation and greater were targeted for serum ELISA and fecal culture testing for MAP. The testing scheme was designed to detect a within-herd prevalence of MAP infection of $2 \%$ or higher with $95 \%$ confidence. Published statistical sample size recommendations, which are based on lactating herd size, were used (USDA-APHIS, 2005). Briefly, all second-lactation and greater cows were tested in herds with fewer than 313 multiparous cows, and in larger herds the number of animals tested increased to a maximum of 360 for fecal culture and 580 for serum ELISA. Milk ELISA was performed on all lactating cows in the herd at the time of DHIA testing.

\section{Sample Collection and Testing Procedures}

Five environmental samples from each operation and paired fecal and serum samples from individual cows were collected by multiple USDA-APHIS-VS personnel on participating farms from March 27 to September 25, 2002. Environmental samples were collected from areas on each operation where manure accumulated from a majority of adult animals. Collectors were instructed to sample common alleyways, manure pits, manure spreaders, lagoons, floors of holding pens, and parlor exits. Environmental samples were collected in $50-\mathrm{mL}$ centrifuge tubes and VS personnel were instructed to fill the tube at least $75 \%$ full $(\sim 37 \mathrm{~mL})$. The protocol for environmental sampling did not specify whether a single sample was to be collected from an area, or whether multiple samples from an area were to be taken and submitted as one sample; consequently, the majority of samples were a single sample representing the area. Environmental, fecal, and serum samples were chilled immediately after collection and shipped on ice to the USDAAPHIS-VS National Veterinary Services Laboratories in Ames, Iowa, where they were stored at $-70,-70$, and $-20^{\circ} \mathrm{C}$, respectively, until tested.

Environmental and individual fecal samples were all cultured by 3 methods [Herrold's egg yolk agar, Becton Dickinson and Company, Franklin Lakes, NJ; BACTEC 460, Becton, Dickinson and Company; and Trek (ESP) culture system II, Trek Diagnostic Systems, Cleveland, $\mathrm{OH}$ ] run in parallel as described previously (Kim et al., 2002; USDA, 2005). Samples that signaled as positive or that had growth on Herrold's egg yolk agar were confirmed as MAP by PCR methods. A sample was reported as positive if it was positive by one or more methods.

All serum samples were tested according to the manufacturer's recommendations, as briefly described by Collins et al. (1993), for antibodies against MAP using a commercially available ELISA (Paracheck; Biocor Ani- mal Health, Omaha, NE), with the exception that samples were tested only in a single well rather than in duplicate. Test results were categorized as negative or positive based on the kit manufacturers' recommendations.

Dairy Herd Improvement Association personnel collected milk samples from all lactating cows, including first-lactation cows, on participating operations during routine milk sampling between June 1, 2002, and January 9, 2003. To preserve milk samples during testing and shipment, they were treated with bronopol (Broad Spectrum Microtabs; D \& F Control Systems, Inc., Dublin, CA). Because collection by USDA personnel and DHIA testing were not coordinated, milk samples were collected from $10 \mathrm{~d}$ prior to $267 \mathrm{~d}$ after serum, fecal, and environmental sample collection.

Milk samples were shipped from multiple DHIA testing laboratories to Antel BioSystems, Inc., in Lansing, Michigan, for testing. The majority of samples were stored frozen $\left(-20^{\circ} \mathrm{C}\right)$, and testing was completed in 1 wk. Individual milk samples were first screened with an indirect ELISA on whole milk samples, and samples reacting in the screening assay ( 0.07 absorbance units above negative controls) were retested in duplicate in an absorbed confirmation assay in routine use on milk samples submitted to Antel Biosystems, Inc. The screening assay has a sensitivity of $99 \%$ and specificity of $62 \%$ compared with the confirmation assay (T. Byrem, Antel Biosystems, Inc., personal communication) and is designed to improve throughput and reduce the cost of analysis of DHIA milk samples compared with the use of the confirmation ELISA alone.

The milk ELISA data set used for the present analysis consisted of sample optical density (OD), negative and positive control OD, and scores (sample OD - negative control OD) from the screening assay on samples that did not react in the initial assay, and of the same information from the confirmation assay on all samples that reacted in the initial assay. Milk ELISA scores greater than the negative control by 0.7 units were considered positive. Any positive test result within a herd, either fecal culture, serum ELISA, or milk ELISA, was used to classify the herd as infected for that particular testing method.

\section{Statistical Analysis}

Descriptive statistics for the environmental samples were produced using SAS (version 8.2, SAS Institute Inc., Cary, NC). The associations between testing positive by environmental culture relative to region; having ever observed cows with clinical JD, defined as chronic diarrhea and weight loss that did not respond to treatment and with a normal appetite; previous testing with an ELISA or fecal culture or both; and herd infection 
Table 1. Number and percentage of individual environmental samples culturing positive for Mycobacterium avium subspecies paratuberculosis by environmental sample source

\begin{tabular}{lcclr}
\hline $\begin{array}{l}\text { Environmental } \\
\text { sample source }\end{array}$ & $\begin{array}{l}\text { Number } \\
\text { positive }\end{array}$ & Total & $\begin{array}{l}\text { Percent } \\
\text { positive }\end{array}$ & SE \\
\hline Parlor exits & 34 & 65 & 52.3 & 6.7 \\
Floors of holding pens & 26 & 53 & 49.1 & 7.4 \\
Common alleyways & 62 & 127 & 48.8 & 5.9 \\
Lagoons & 18 & 38 & 47.4 & 9.1 \\
Manure spreaders & 11 & 26 & 42.3 & 11.6 \\
Manure pits & 17 & 41 & 41.5 & 8.7 \\
Other & 50 & 133 & 37.6 & 5.6 \\
Total & 218 & 483 & 45.1 & 4.0 \\
\hline
\end{tabular}

status, determined by individual animal sampling using fecal culture, serum ELISA, and milk ELISA, were evaluated using a log likelihood $\chi^{2}$ test of association or Fisher's exact test where appropriate. Individual sample analysis was done using statistical software (SUDAAN, Release 9.0, 2004; Research Triangle Institute, Research Triangle Park, NC) that accounted for the nonindependence of samples through a nested analysis. The Cochran-Armitage trend test was used to evaluate the association between testing positive with environmental cultures and herd size and a categorized RHA milk production. The correlation between the percentage of positive environmental samples and the within-herd prevalence, as determined by each individual testing method, was evaluated using Spearman's rank correlation test. $P$-values of $\leq 0.05$ were considered significant.

\section{RESULTS}

\section{Sample Level}

Sample Area. Environmental sampling was conducted by 85 USDA-APHIS-VS personnel on 98 herds participating in the Dairy 2002 survey. A total of 483 samples were collected and cultured, and of these, 218 (45.1\%) were culture-positive for MAP (Table 1). Five environmental samples were collected from 91 operations, whereas only 4 environmental samples were collected on 7 operations. Similar percentages of positive environmental cultures were collected from all designated areas [parlor exits (52.3\%), floors of holding pens (49.1\%), common alleyways (48.8\%), lagoons (47.4\%), manure spreaders $(42.3 \%)$, and manure pits $(41.5 \%)$ ]. There was no association between source of the environmental sample and culture result $(P=0.63)$.

The largest number of samples was collected from common alleyways $(26.3 \%, 127 / 483)$ and "other" areas $(25.5 \%, 133 / 483)$. Areas sampled and labeled as "other" were numerous and included barn cleaners, gutters, dry lots, chute areas, and sick pens.

Month of Sampling. The majority of environmental samples were collected between April and July and are shown in Table 2. When more than 20 samples were collected during a month, the percentage of samples that were culture-positive ranged from 32.8 to $60.6 \%$. There was not a significant association between month of sampling and environmental results $(P=0.18)$ after controlling for clustering at the operation level.

\section{Operation Level}

Of the 98 operations participating in the study, 69 (70.4\%) had at least one culture-positive environmental sample. Of the operations with positive environmental samples, 15, 10, 12, 11, and 21 operations had 1, 2, 3,4 , and 5 culture-positive samples, respectively. The percentage of operations that were culture-positive was similar across all sampling sites on operations and ranged from $42.9 \%$ of operations with samples from manure spreaders to $55.4 \%$ of operations with samples from parlor exits (Table 3 ). There was no association between environmental sample source and culture result at the operation level $(P=0.94)$.

Herd Size. A similar percentage of operations among herd size categories had at least one culture-positive environmental sample for MAP: $66.7 \%$ (26/39), $69.7 \%$ (23/33), and 76.9\% (20/26) for small, medium, and large herds, respectively. There was no association between herd size and environmental culture result $(P=0.67)$ and no trend was observed $(P=0.385)$.

Region. A significant association was observed between environmental culture results and region at the operation level (Fisher's exact test, $P=0.017$ ). The Midwest region had the largest percentage of operations with positive environmental cultures (83.3\%), whereas the Northeast region had the lowest percentage (43.8; Table 4).

Month of Sampling. The number of operations that had at least one culture-positive environmental sample was greatest in May (17/19), whereas it was lowest in September (1/3). There was not a significant association between month of sampling and the operation having at least one positive environmental sample (Fisher's exact test, $P=0.054$; Table 5).

RHA Milk Production. Only one (6.25\%) operation on which the RHA milk production was less than 7,000 $\mathrm{kg}$ was environmental culture-negative, whereas 8 (50.0\%) operations on which the RHA milk production was $11,000 \mathrm{~kg}$ or more were culture-negative (Figure 1). A significant association was found between categorized RHA milk production and environmental culture results (Fisher's exact test, $P=0.048$ ) and a significant trend was found with a negative association between milk production and the operation having a positive environmental sample result (Cochran-Armitage trend test, $P=$ 0.004). 
Table 2. Number of individual environmental samples by culture result for Mycobacterium avium subspecies paratuberculosis and month of collection

\begin{tabular}{lccccccc}
\hline \multirow{2}{*}{$\begin{array}{l}\text { Environmental } \\
\text { sample result }\end{array}$} & March & April & May & June & July & August & September \\
\cline { 2 - 8 } & 6 & 44 & 57 & 42 & 51 & 13 & 5 \\
Positive & 9 & 90 & 37 & 68 & 39 & 12 & 10 \\
Negative & 15 & 134 & 94 & 110 & 90 & 25 & 15 \\
Total & 15 & &
\end{tabular}

Housing. There was no association between housing type and environmental sample result (Fisher's exact test, $P=0.147$ ). All operations reporting an absence of housing facilities (no physical structures) for lactating cows $(n=7)$ were environmental culture-positive for MAP. Similar percentages of operations with single $(\mathrm{n}=$ $26)$ and multiple $(\mathrm{n}=65)$ animal housing were environmental culture-positive (61.5 and $70.8 \%$, respectively).

JD Knowledge. There was a significant association between producer knowledge of JD and environmental sample results (Fisher's exact test, $P<0.001$; Table 6). Although both operations that had not heard of the disease were environmental culture-positive, a significant trend was observed, with increasing producer knowledge associated with an increased percentage of operations with positive environmental cultures (Cochran-Armitage trend test, $P<0.001$ ).

JD History. Fifty of 58 herds $(86.2 \%)$ that had ever observed cows with clinical signs consistent with JD had at least one positive environmental sample, whereas $85.4 \%$ (41/48) of farms that had observed cows with signs of JD in the past 12 mo were environmental samplepositive (Table 7). A highly significant association $(P<$ 0.001 ) was observed between operations testing positive by environmental culture and operations on which cows had ever been observed with clinical signs. Observing clinical signs in the 12 mo prior to the study was also significantly associated with operations testing positive by environmental culture $(P<0.001)$. There was no significant association between environmental culture results and previous testing for MAP infection using either

Table 3. Number and percentage of operations testing culture-positive for Mycobacterium avium subspecies paratuberculosis by source of environmental sample

\begin{tabular}{llllr}
\hline $\begin{array}{l}\text { Environmental } \\
\text { sample source }\end{array}$ & $\begin{array}{l}\text { Operations } \\
\text { positive }\end{array}$ & Total & $\begin{array}{l}\text { Percent } \\
\text { positive }\end{array}$ & SE \\
\hline Parlor exits & 31 & 56 & 55.4 & 6.7 \\
Common alleyways & 35 & 71 & 49.3 & 5.9 \\
Lagoons & 16 & 33 & 48.5 & 8.7 \\
Floors of holding pens & 23 & 49 & 46.9 & 7.1 \\
Manure pits & 16 & 36 & 44.4 & 8.3 \\
Manure spreaders & 9 & 21 & 42.9 & 10.8 \\
Other & 33 & 64 & 51.6 & 6.7 \\
Any source & 69 & 98 & 70.4 & 4.6 \\
\hline
\end{tabular}

serum ELISA or fecal culture in the 12 mo prior to the study $[P=0.074$ and 0.714 (Fisher's exact test), respectively].

Comparison with Individual Animal Testing. Of the 98 operations from which environmental samples were cultured, individual animal fecal culture, serum ELISA, and milk ELISA results were available for 60, 97, and 34 operations, respectively (Table 8 ). There was a significant association between herds that were environmental culture-positive and those having at least one animal testing fecal culture-positive (Fisher's exact test, $P=0.001$ ). Fifty of the 60 herds sampled by fecal culture had at least one positive individual culture and were determined to be infected. Environmental sampling identified $38(76.0 \%)$ of the 50 infected operations, and 2 of the 10 operations classified as not infected based on individual animal fecal culture had one culture-positive environmental sample.

Of the 12 herds that were positive by individual fecal culture and negative by environmental culture, 58.3\% had within-herd fecal culture prevalence estimates of less than $5 \%$, and 5 operations had an apparent prevalence of $2.6 \%$ or less. Three environmental samples from one of the 12 herds were mistakenly collected from calf pens, rather than adult areas, and this herd had the highest within-herd fecal culture prevalence $(21.9 \%)$ of those operations not identified by environmental culture. Nine of the 12 operations reported never having observed a cow with clinical signs of JD. Eight of the 12 operations were classified as infected based on serum ELISA testing.

Both of the fecal culture-negative, environmental culture-positive herds were small Midwest herds. Nineteen individual fecal samples and 5 environmental samples

Table 4. Operation-level environmental culture results for Mycobacterium avium subspecies paratuberculosis by region of the United States

\begin{tabular}{lllcc}
\hline & \multicolumn{4}{c}{ Region } \\
\cline { 2 - 5 } Environmental & West & Midwest & Northeast & Southeast \\
\hline Positive & 16 & 35 & 7 & 11 \\
Negative & 10 & 7 & 9 & 3 \\
Total & 26 & 42 & 16 & 14 \\
\hline
\end{tabular}


Table 5. Operation-level environmental culture results for Mycobacterium avium subspecies paratuberculosis by month of collection

\begin{tabular}{llllllll}
\hline & \multicolumn{7}{c}{ Month of collection } \\
\cline { 2 - 8 } Environmental & March & April & May & June & July & August & September \\
\hline Positive & 2 & 14 & 17 & 16 & 15 & 4 & 1 \\
Negative & 1 & 13 & 2 & 7 & 3 & 1 & 2 \\
Total & 3 & 27 & 19 & 23 & 18 & 5 & 3 \\
\hline
\end{tabular}

were cultured from one herd (herd A) in which the only MAP-positive environmental sample was obtained from unspecified heifer housing. Fifty-four fecal samples and 5 environmental samples were collected and cultured from a second operation (herd B), with the single MAPpositive environmental sample coming from the manure pit.

Eighty operations were classified as infected based on at least one serum ELISA-positive test result and of these, $61(76.3 \%)$ were identified as positive by environmental culture. Seven of 17 operations (41.2\%) classified as not infected by serum ELISA were environmental culture-positive. The association between environmental culture and serum ELISA results was significant $(P=$ 0.006 ).

No significant association was found between infected operations based on milk ELISA and those tested by environmental culture (Fisher's exact test, $P=0.154$ ). A total of 34 operations were tested with both environmental culture and milk ELISA. Twenty of $28(71.4 \%)$ operations identified as infected based on milk ELISA were environmental culture-positive. Two of 6 operations classified as not infected by milk ELISA were environmental culture-positive.

A significant positive correlation was identified between the percentage of positive environmental samples and the within-herd prevalence estimates determined by individual animal testing $(P<0.001)$. Spearman's rank correlation coefficients were $0.685,0.531$, and 0.648

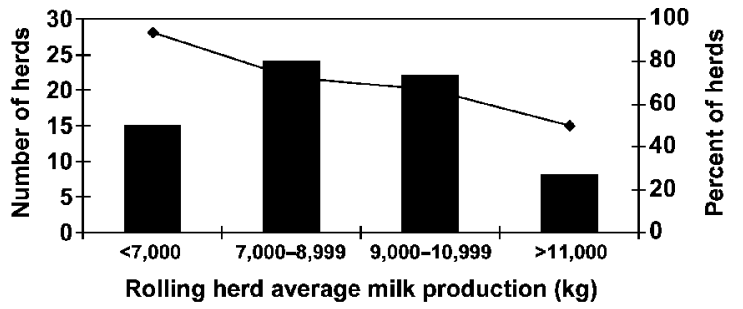

Number of culture-positive herds $\rightarrow \bullet$ Percent culture-positive herds

Figure 1. Frequency and percentage of herds with at least one culture-positive environmental sample for Mycobacterium avium subspecies paratuberculosis by rolling herd average milk production during 2001. for fecal culture, serum ELISA, and milk ELISA estimates, respectively.

\section{DISCUSSION}

This is the first nationwide study evaluating the distribution of MAP in the environment of US dairy farms and the feasibility of using environmental culture to determine herd infection status. The study evaluated herds of different sizes and with different histories of JD, from different regions of the country. Although operations were chosen to represent high and low risk of MAP infection, we believe the results from this study represent the typical distribution of MAP in a dairy farm environment. However, caution should be used in extending the operation-level estimates to the US dairy population because the herds in this study were not randomly selected.

Testing programs designed to classify herds initially as uninfected or low risk currently use serum ELISA and fecal culture testing in series. Serum ELISA sensitivity is poor, however, and many subclinical cows may test negative by this method, leading to low-prevalence herds falsely declared as uninfected. However, individual fecal culture testing, the most sensitive noninvasive method available, is expensive and may require 3 to 4 mo to obtain results. Under the voluntary program, animals that test ELISA-positive can have fecal cultures performed to confirm disease. Because the sensitivity of fecal culture is also less than $100 \%$, as reported by Whitlock et al. (2000), infected animals may be serum ELISA-positive and fecal culture-negative, leading the herd to be declared not infected. Serum ELISA and indi-

Table 6. Producer knowledge of Johne's disease by environmental culture results for Mycobacterium avium subspecies paratuberculosis

\begin{tabular}{lccc}
\hline & \multicolumn{3}{c}{$\begin{array}{c}\text { Operation environmental } \\
\text { result }\end{array}$} \\
\cline { 2 - 4 } Producer knowledge & Positive & Negative & Total \\
\hline of Johne's disease & 2 & 0 & 2 \\
Had not heard of before & 2 & 7 & 9 \\
Recognized name only & 18 & 15 & 33 \\
Knew some basics & 46 & 7 & 53 \\
Fairly knowledgeable & &
\end{tabular}


Table 7. Operation environmental sample result for Mycobacterium avium subspecies paratuberculosis by history of Johne's disease occurrence and testing

\begin{tabular}{|c|c|c|c|c|c|c|c|c|}
\hline \multirow{3}{*}{$\begin{array}{l}\text { Operation } \\
\text { environmental } \\
\text { result }\end{array}$} & \multicolumn{8}{|c|}{$\begin{array}{c}\text { Operation history } \\
\text { of Johne's disease and testing }\end{array}$} \\
\hline & \multicolumn{2}{|c|}{$\begin{array}{l}\text { Ever observed } \\
\text { clinical signs } \\
\quad(\mathrm{n}=91)\end{array}$} & \multicolumn{2}{|c|}{$\begin{array}{l}\text { Clinical signs } \\
\text { observed }^{1} \\
(\mathrm{n}=90)\end{array}$} & \multicolumn{2}{|c|}{$\begin{array}{c}\text { Serum ELISA } \\
\text { testing } \\
(\mathrm{n}=89)\end{array}$} & \multicolumn{2}{|c|}{$\begin{array}{c}\text { Fecal culture } \\
\text { testing }{ }^{1} \\
(\mathrm{n}=89)\end{array}$} \\
\hline & Yes & No & Yes & No & Yes & No & Yes & No \\
\hline Positive & 50 & 13 & 41 & 21 & 22 & 39 & 7 & 54 \\
\hline Negative & 8 & 20 & 7 & 21 & 5 & 23 & 2 & 26 \\
\hline Total & 58 & 33 & 48 & 42 & 27 & 62 & 9 & 80 \\
\hline
\end{tabular}

${ }^{1}$ In 12 mo prior to the interview.

vidual fecal culture sampling also require handling individual cattle, which is time-consuming. Therefore, a method with similar sensitivity to individual animal sampling that is less intrusive and less expensive is preferable.

The results of this study suggest that individual animal sampling can be replaced by environmental sampling to determine herd infection status. Targeted sampling and culture of common alleyways, milking parlor exits, gutters and gutter cleaners, and lagoons or manure pits can assess herd infection status with reasonable reliability. Environmental sampling provides a convenient and affordable alternative strategy for herd screening, and may eventually replace serum ELISA as an initial herd-level screening process, as described by Berghaus et al. (2006).

Environmental manure samples for culture may prove to be a less expensive method for determining herd infection status. Raizman et al. (2004) estimated that it takes 30 min of sampling and $\$ 100$ of laboratory fees to determine herd status with approximately $90 \%$ accuracy by environmental testing, as opposed to at least $\$ 180$ of laboratory fees and additional time and veterinary labor fees for serum ELISA testing. The current study found herd-level environmental culture sensitivity greater than $70 \%$ when compared with either whole-herd or statistical-subset testing of individual animals by fecal culture, serum ELISA, or milk ELISA, confirming an acceptable accuracy level for environmental sampling. Re- sults from Raizman et al. (2004) and this study were used in deciding to implement environmental sampling and culture as a means of obtaining level 1 of the testnegative component in the US Voluntary Bovine Johne's Disease Control Program (USDA-APHIS, 2005). However, the national program requires 6 environmental samples rather than the 5 reported in this study.

Even though multiple personnel were involved in obtaining environmental samples from a variety of operations, the results are similar to those reported by Raizman et al. (2004) and Berghaus et al. (2006). These are important variables to consider when implementing a national control program in which multiple people are involved in sample collection. Thus, personnel administering JD control programs should seriously consider the advantages of environmental culture to determine herd status for MAP.

Because environmental samples are collected from areas where a large proportion of cows defecate on a daily basis, with the exception of lagoons and manure pits, the weather or season of collection was not expected to affect the isolation of the organism, and no difference was observed in this study. Mycobacterium avium subspecies paratuberculosis has been shown to remain viable in the environment for long periods of time, so even when sampling lagoons and manure pits, the potential for finding the organism, if present, is good. As a tool to be used in the national program, it appears that environ-

Table 8. Comparison of operation-level environmental and individual sample test results for Mycobacterium avium subspecies paratuberculosis

\begin{tabular}{|c|c|c|c|c|c|c|}
\hline \multirow{3}{*}{$\begin{array}{l}\text { Environmental } \\
\text { sample result }\end{array}$} & \multicolumn{6}{|c|}{ Individual sample } \\
\hline & \multicolumn{2}{|c|}{ Fecal culture $(\mathrm{n}=60)$} & \multicolumn{2}{|c|}{ Serum ELISA $(\mathrm{n}=97)$} & \multicolumn{2}{|c|}{ Milk ELISA $(\mathrm{n}=34)$} \\
\hline & Positive & Negative & Positive & Negative & Positive & Negative \\
\hline Positive & 38 & 2 & 61 & 7 & 20 & 2 \\
\hline Negative & 12 & 8 & 19 & 10 & 8 & 4 \\
\hline Total & 50 & 10 & 80 & 17 & 28 & 6 \\
\hline
\end{tabular}


mental samples could be obtained at any time of the year without affecting the sensitivity of the method.

There was a significant difference in the percentage of operations that tested environmental culture-positive in different regions of the country, with the Midwest being the highest and Northeast the lowest. The herd selection process apparently does not account for this difference, as there were no associations between region and herd-level prevalence by fecal culture, serum ELISA, or milk ELISA. Although this may have something to do with location, temperature, humidity, and the like, it is also possible that the herd-level prevalence of MAP was different among regions, as reported by Wells and Wagner (2000).

Multiple studies have reported that cows testing positive for MAP produce less milk than test-negative cows (Nordlund et al., 1996; Hendrick et al., 2005; Lombard et al., 2005). This study found that herd-level milk production was negatively associated with MAP infection. Although the disease may be responsible for part of the reduced production, it is also possible that management practices that result in low milk production, with the exception of those in grazing herds, may also influence MAP transmission. The herd selection process did not appear to bias this result, because there was no association between assigned herd risk and RHA.

The ability of environmental testing to establish the presence of MAP in manure spreaders, manure piles, and lagoons is applicable in other ways by farms that use manure as fertilizer. As part of a farm's JD control strategy, preventing the spread of MAP over fields used either as pasture or to grow crops should be a priority. In the Dairy 2002 study (USDA, 2002), it was determined that between 40 and $50 \%$ of farms spread manure on forage ground grazed or harvested for cattle or calves. This study found that $42 \%$ (11/32) of manure spreaders tested were culture-positive for MAP compared with 7\% in the Raizman et al. (2004) study, where 80 known infected herds and 28 herds thought to be noninfected were evaluated. The potential of transmitting MAP infection through feed contamination subsequent to field contamination should be seriously considered.

On 2 operations individual fecal samples were all culture-negative, but each operation had one culture-positive environmental sample. Only one first-lactation animal was sampled from either farm and she was from herd A. This herd also had no animals testing serum ELISA-positive. Herd A had purchased 2 lactating cows in the prior year. Herd B had 2 animals that were serum ELISA-positive and one that was inconclusive. No animals were imported during the previous year. On neither operation had cows ever been observed with clinical signs of JD or had any testing been done for MAP infection. Because not all cows in either herd were tested (herd A:
25 cows; herd B: 71 cows), it is possible that nontested cows (primarily first-lactation animals) were shedding MAP. In herd A, where the heifer pen was culture-positive, younger animals appeared to be infected and shedding the organism. In herd B, where the manure pit was culture-positive, it was also possible that the shedding animal had already been removed from the operation prior to becoming clinically affected.

Previous studies (Raizman et al., 2004; Berghaus et al., 2006) evaluating environmental sampling have reported a correlation between individual or pooled animal prevalence estimates and the prevalence of environmental culture, with similar results as those found in this study. Raizman et al. modeled environmental prevalence using fecal pools to estimate within-herd prevalence for MAP. Although there was a strong correlation between the prevalence estimates obtained from either method, the potential for individual animals shedding extremely large amounts of MAP within a herd, as described by Whitlock et al. (2005), may lead to overestimation of the true within-herd prevalence.

In contrast to the 2 previous studies evaluating environmental sampling, individual samples in this study were collected at the same time and cultured as individual samples, not fecal pools. With the exception of milk samples, all data used in this analysis from an individual farm were collected at one time. Although 3 culture methods were used, which likely increased the sensitivity of the method, the same methods were used for both environmental and individual fecal samples.

It is possible that the operations used in this study had an increased awareness of JD, because they chose to participate. However, in $36.3 \%$ (33/91) of the herds surveyed, cattle with clinical signs consistent with JD had never been observed, but MAP was cultured from the environment of $39.4 \%$ (13/33) of those herds. Operations with increased knowledge of the disease may have made management changes that limited environmental contamination (removing heavy MAP-shedding cows), had low within-herd prevalence levels, and were not always detected by environmental cultures. Two producers that had not heard of JD had environmental samples that cultured positive, suggesting that clinical disease was not occurring, or at least was not recognized in those herds.

Another possible limitation to this study is the sensitivity of the fecal culture. It is possible, with the constraints on fecal culture sensitivity and the inability to take fecal culture samples from every cow on every farm, that some herds were mistakenly identified as not infected. Farms with low prevalence and light-shedding cows, especially if some cows that were shedding were not sampled, could fail to detect MAP by fecal culture. This is underscored by the 2 farms that were negative 
by individual fecal culture but positive by environmental culture. Accuracy when determining the sensitivity of a test is limited by the sensitivity of the comparison test, in this case the imperfect fecal culture.

The environmental sampling method used in this study was a form of pooling. The method involved taking environmental samples of feces, which likely contained material from several cows, from 5 locations on each farm. Samples collected from operations with low withinherd prevalence of MAP and low levels of MAP in the environment may not be detected as positive with this method because of bacterial numbers falling below the sensitivity threshold of the culture method.

\section{CONCLUSIONS}

Environmental sampling to determine herd infection status requires less labor and time than individual cow sampling and is equivalent in cost to performing serum ELISA on 30 animals. Environmental sampling should be instituted on dairy operations to determine and monitor herd status for MAP and can be used to target areas on the operation that pose the greatest risk of environmental exposure to MAP.

\section{REFERENCES}

Berghaus, R. D., T. B. Farver, R. J. Anderson, C. C. Jaravata, and I. A. Gardner. 2006. Environmental sampling for detection of Mycobacterium avium ssp. paratuberculosis on large California dairies. J. Dairy Sci. 89:963-970.

Collins, M. T., A. Angulo, C. D. Buergelt, S. G. Hennager, S. K. Hietala, R. H. Jacobson, D. L. Whipple, and R. H. Whitlock. 1993. Reproducibility of a commercial enzyme-linked immunosorbent assay for bovine paratuberculosis among eight laboratories. J. Vet. Diagn. Invest. 5:52-55.

Hendrick, S. H., D. F. Kelton, K. E. Leslie, K. D. Lissemore, M. Archambault, and T. F. Duffield. 2005. Effects of paratuberculosis on culling, milk production, and milk quality in dairy herds. J. Am. Vet. Med. Assoc. 227:1302-1308.

Jorgensen, J. B. 1977. Survival of Mycobacterium paratuberculosis in slurry. Nord. Vet. Med. 29:267-270.

Kalis, C. H., J. W. Hesselink, H. W. Barkema, and M. T. Collins. 2000. Culture of strategically pooled bovine fecal samples as a method to screen herds for paratuberculosis. J. Vet. Diagn. Invest. 12:547-551.

Kennedy, D. J., and G. Benedictus. 2001. Control of Mycobacterium avium subsp. paratuberculosis infection in agricultural species. Rev. Sci. Tech. 20:151-179.

Kim, S. G., S. J. Shin, R. H. Jacobson, L. J. Miller, P. R. Harpending, S. M. Stehman, C. A. Rossiter, and D. A. Lein. 2002. Development and application of quantitative polymerase chain reaction assay based on the ABI 7700 system (TaqMan) for detection and quantification of Mycobacterium avium subsp. paratuberculosis. J. Vet. Diagn. Invest. 14:126-131.
Lombard, J. E., F. B. Garry, B. J. McCluskey, and B. A. Wagner. 2005. Risk of removal and effects on milk production associated with paratuberculosis status in dairy cows. J. Am. Vet. Med. Assoc. 227:1975-1981.

Nordlund, K. V., W. J. Goodger, J. Pelletier, and M. T. Collins. 1996. Associations between subclinical paratuberculosis and milk production, milk components, and somatic cell counts in dairy herds. J. Am. Vet. Med. Assoc. 208:1872-1876.

Raizman, E. A., S. J. Wells, S. M. Godden, R. F. Bey, M. J. Oakes, D. C. Bentley, and K. E. Olsen. 2004. The distribution of Mycobacterium avium ssp. paratuberculosis in the environment surrounding Minnesota dairy farms. J. Dairy Sci. 87:2959-2966.

Sockett, D. C. 1996. Johne's disease eradication and control: Regulatory implications. Vet. Clin. North Am. Food Anim. Pract. 12:431-440.

Sweeney, R. W. 1996. Transmission of paratuberculosis. Vet. Clin. North Am. Food Anim. Pract. 12:305-312.

Tavornpanich, S., I. A. Gardner, R. J. Anderson, S. Shin, R. H. Whitlock, T. Fyock, J. M. Adaska, R. L. Walker, and S. K. Hietala. 2004. Evaluation of microbial culture of pooled fecal samples for detection of Mycobacterium avium subsp. paratuberculosis in large dairy herds. Am. J. Vet. Res. 65:1061-1070.

USDA. 2005. Johne's Disease on U.S. Dairy Operations, 2002. USDAAPHIS (Animal and Plant Health Inspection Service)-VS, Centers for Epidemiology and Animal Health, National Animal Health Monitoring System, Fort Collins, CO.

USDA. 2002. Part III: Reference of Dairy Cattle Health and Health Management Practices in the United States. USDA-APHIS (Animal and Plant Health Inspection Service)-VS, Centers for Epidemiology and Animal Health, National Animal Health Monitoring System, Fort Collins, CO.

USDA-APHIS (Animal and Plant Health Inspection Service). 2005. Uniform Program Standards for the Voluntary Bovine Johne's Disease Control Program. APHIS Publ. 91-45-016. Ruminant Health Programs, National Center for Animal Health Programs, USDA-APHIS-VS, Riverdale, MD.

van Schaik, G., S. M. Stehman, Y. H. Schukken, C. R. Rossiter, and S. J. Shin. 2003. Pooled fecal culture sampling for Mycobacterium avium subsp. paratuberculosis at different herd sizes and prevalence. J. Vet. Diagn. Invest. 15:233-241.

Wells, S. J., S. M. Godden, C. J. Lindeman, and J. E. Collins. 2003. Evaluation of bacteriologic culture of individual and pooled fecal samples for detection of Mycobacterium paratuberculosis in dairy cattle herds. J. Am. Vet. Med. Assoc. 223:1022-1025.

Wells, S. J., and B. A. Wagner. 2000. Herd-level risk factors for infection with Mycobacterium paratuberculosis in US dairies and association between familiarity of the herd manager with the disease or prior diagnosis of the disease in that herd and use of preventive measures. J. Am. Vet. Med. Assoc. 216:1450-1457.

Wells, S. J., R. H. Whitlock, C. J. Lindeman, and T. Fyock. 2002. Evaluation of bacteriologic culture of pooled fecal samples for detection of Mycobacterium paratuberculosis. Am. J. Vet. Res. 63:1207-1211.

Whitlock, R. H., S. J. Wells, R. W. Sweeney, and J. Van Tiem. 2000. ELISA and fecal culture for paratuberculosis (Johne's disease): Sensitivity and specificity of each method. Vet. Microbiol. 77:387-398.

Whitlock, R. H., R. W. Sweeney, T. L. Fyock, and J. Smith. 2005. MAP super-shedders: Another factor in the control of Johne's disease. Page 164 in 8th Intl. Colloq. Paratb. Proc., Copenhagen, Denmark. E. J. B. Manning and S. S. Nielsen, ed. Intl. Assoc. Paratuberculosis, Madison, WI.

Whittington, R. J., D. J. Marshall, P. J. Nicholls, I. B. Marsh, and L. A. Reddacliff. 2004. Survival and dormancy of Mycobacterium avium subsp. paratuberculosis in the environment. Appl. Environ. Microbiol. 70:2989-3004. 